Supplement of Hydrol. Earth Syst. Sci., 22, 6163-6185, 2018

https://doi.org/10.5194/hess-22-6163-2018-supplement

(C) Author(s) 2018. This work is distributed under

the Creative Commons Attribution 4.0 License.

(c) (1)

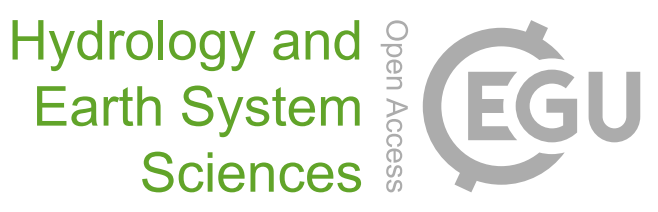

Supplement of

\title{
Toward a conceptual framework of hyporheic exchange across spatial scales
}

\section{Chiara Magliozzi et al.}

Correspondence to: Robert C. Grabowski (r.c.grabowski@cranfield.ac.uk)

The copyright of individual parts of the supplement might differ from the CC BY 4.0 License. 
Table s1: Collection of worldwide field studies on the hyporheic exchange across a broad range of hydrogeological, topographical and geological settings. Table shows references, geographical zone and state, river name, and information on catchment scale: geology (sediment size and hydraulic conductivity), topographical/morphological, ecological (in-channel vegetation), anthropogenic (agricultural or human infrastructure).

\begin{tabular}{|c|c|c|c|c|}
\hline Source & Hydrological & Hydrogeological & Topographical & Anthropogenic \& Ecological \\
\hline $\begin{array}{l}\text { Angermann et al., } \\
2012\end{array}$ & $\begin{array}{l}\text { Europe, UK; Atlantic biogeographic } \\
\text { region; River Tern; Rainfall: } 583 \text { mm / } \\
\text { yr }\end{array}$ & $\begin{array}{l}\text { Dominant geology is } \\
\text { Permo-Triassic sandstone }\end{array}$ & 1 & 1 \\
\hline $\begin{array}{l}\text { Anibas et al., } \\
2012\end{array}$ & $\begin{array}{l}\text { Europe;Poland; Continental } \\
\text { biogeographic region. Rainfall: } 550- \\
700 \mathrm{~mm} / \mathrm{yr}, 3 \text { subcatchments }\end{array}$ & $\begin{array}{l}\text { Extensive depression } \\
\text { formed during the last } \\
\text { glaciations. } \\
\text { Unconsolidated aquifers } \\
\text { intermixed with confined } \\
\text { ones }\end{array}$ & / & $\begin{array}{l}\text { mostly natural but in the low areas of } \\
\text { the catchment meadows and } \\
\text { pastures }\end{array}$ \\
\hline $\begin{array}{l}\text { Anibas et al., } \\
2009\end{array}$ & $\begin{array}{l}\text { Europe; Belgium; Continental } \\
\text { biogeographic region }\end{array}$ & / & I & $\begin{array}{l}\text { agricultural landuse and weirs } \\
\text { structures }\end{array}$ \\
\hline $\begin{array}{l}\text { Arntzen et al., } \\
2006\end{array}$ & $\begin{array}{l}\text { USA; Washington; Columbia Rive; } \\
\text { Hanford Reachr; Continental } \\
\text { biogeographic region }\end{array}$ & $\begin{array}{l}\text { Miocene to Pliocene of } \\
\text { the Ringold Formation, } \\
\text { Pleistocene flood gravels } \\
\text { of the Hanford Formation }\end{array}$ & / & / \\
\hline $\begin{array}{l}\text { Bourke et al., } \\
2014\end{array}$ & $\begin{array}{l}\text { Australia; Subtropical Biogeographic } \\
\text { region; Marillana Creek }\end{array}$ & $\begin{array}{l}\text { pisolitic goethite, chert } \\
\text { and dolerite }\end{array}$ & / & mine \\
\hline Briggs et al., 2010 & $\begin{array}{l}\text { USA; Massachussets; Ipswich River; } \\
\text { humid continental biogeographic } \\
\text { region }\end{array}$ & / & I & three main stem anthropogenic dams \\
\hline $\begin{array}{l}\text { Czernuszenko et. } \\
\text { al., } 1998\end{array}$ & $\begin{array}{l}\text { Europe;Moldavia; Botna,Byk and } \\
\text { Kogilnik Rivers; Continantal } \\
\text { biogeographic region }\end{array}$ & / & / & / \\
\hline Datry et al 2008 & $\begin{array}{l}\text { New Zealand; Selwyn River,Atlantic } \\
\text { biogeographic region }\end{array}$ & $\begin{array}{l}\text { confined and semi- } \\
\text { confined aquifers }\end{array}$ & I & / \\
\hline $\begin{array}{l}\text { Dujardin et al } \\
2014\end{array}$ & $\begin{array}{l}\text { Europe; Belgium; Continental } \\
\text { biogeographic region ;Zenne River } \\
\text { Rainfall: } 852 \mathrm{~mm} / \text { year }\end{array}$ & / & / & $\begin{array}{l}\text { considerable chemical industrial } \\
\text { activity }\end{array}$ \\
\hline Duke et al 2007 & $\begin{array}{l}\text { USA; Texas;Cow Bayou Stream; } \\
\text { Humid Subtropical biogeographic } \\
\text { region. Rainfall: } 82 \mathrm{~cm} / \mathrm{yr}\end{array}$ & Eagle Ford shale & I & $\begin{array}{l}\text { Dam structure. Agriculture and } \\
\text { rangeland Small riparian forest of } \\
\text { Ulmus crassifolia, Fraxinus }\end{array}$ \\
\hline
\end{tabular}




\begin{tabular}{|c|c|c|c|c|}
\hline & & & & texensis, Juniperus ashei \\
\hline $\begin{array}{l}\text { Edwardson et al., } \\
2003\end{array}$ & $\begin{array}{l}\text { USA;Alaska;Kuparuk River; Oceanic- } \\
\text { Artic biogeographic region }\end{array}$ & 1 & 1 & 1 \\
\hline $\begin{array}{l}\text { Fernald et al., } \\
2001\end{array}$ & $\begin{array}{l}\text { USA; Oregon;Willamette River; Humid- } \\
\text { semiarid biogeographic region }\end{array}$ & Holocene alluvium & 1 & 1 \\
\hline $\begin{array}{l}\text { Gooseff et al., } \\
2003\end{array}$ & $\begin{array}{l}\text { Antartica; Delta Stream and Green } \\
\text { Creek; Polar biogeographic region }\end{array}$ & 1 & 1 & 1 \\
\hline $\begin{array}{l}\text { Haggard et al., } \\
2001\end{array}$ & $\begin{array}{l}\text { USA; Oklahoma; Dry Creek, Cloud } \\
\text { Creek, and Cherokee Creek (Lake } \\
\text { Eucha-Spavinaw Basin); humid- } \\
\text { subtropical }\end{array}$ & karstic & 1 & 1 \\
\hline Hall et al., 2002 & $\begin{array}{l}\text { USA; New Hampshire; } 13 \text { Streams; } \\
\text { Continantal Biogeographic region }\end{array}$ & bedrock superficial & & $\begin{array}{l}\text { forested: Fagus grandifolia, Acer } \\
\text { saccharumBetula alleghaniensis }\end{array}$ \\
\hline Hart et el., 1999 & $\begin{array}{l}\text { USA; Tennessee; West Fork of Walker } \\
\text { Branch; Continental biogeographic } \\
\text { region }\end{array}$ & bedrock outcrops & 1 & I \\
\hline $\begin{array}{l}\text { Harvey and Fuller, } \\
1998 \\
\text { Harvey et al. } \\
2003\end{array}$ & $\begin{array}{l}\text { USA; Arizona; Pinal Creek; semi- } \\
\text { desert biogeographic region }\end{array}$ & $\begin{array}{l}\text { regional aquifer } \\
\text { composed of partially } \\
\text { cemented basin fill, a } \\
\text { more shallow one is } \\
\text { present (sand and } \\
\text { gravel). The aquifer } \\
\text { presents igneous rock } \\
\text { formations and can be } \\
\text { constricted }\end{array}$ & 1 & I \\
\hline Jones et al. , 2008 & $\begin{array}{l}\text { USA; Oregon; Umatilla River; humid- } \\
\text { semidesertic biogeographic region }\end{array}$ & Columbia Plateau basalt & $\begin{array}{l}\text { naturally } \\
\text { anabranched }\end{array}$ & 1 \\
\hline $\begin{array}{l}\text { Kasahara et al., } \\
2003\end{array}$ & $\begin{array}{l}\text { USA; Oregon; Lookout Creek;Humid- } \\
\text { semiarid, biogeographic region. } \\
\text { Rainfall:2300 }-3550 \mathrm{~mm} / \mathrm{yr}\end{array}$ & 1 & 1 & $\begin{array}{l}\text { forested Psudotsuga menziesii, } \\
\text { Tsuga heterophylla, Thuja plicata, } \\
\text { Alnus rubra and Salix spp. }\end{array}$ \\
\hline
\end{tabular}




\begin{tabular}{|c|c|c|c|c|}
\hline $\begin{array}{l}\text { Kasahara et al. , } \\
2006\end{array}$ & $\begin{array}{l}\text { Canada; Ontario; Rouge River tributary } \\
\text { 1; Rouge River tributary 2; Silver } \\
\text { Creek; Continental biogeographic } \\
\text { region }\end{array}$ & unconfined aquifer & 1 & l \\
\hline $\begin{array}{l}\text { Kasahara et al., } \\
2007\end{array}$ & $\begin{array}{l}\text { Canada; Ontario; Boyne River and } \\
\text { others; Continental biogeographic } \\
\text { region }\end{array}$ & 1 & 1 & 1 \\
\hline Kaser et al., 2013 & $\begin{array}{l}\text { Europe; UK; River Leith; Atlantic } \\
\text { biogeographic. Rainfall } 900 \text { mm/yr }\end{array}$ & $\begin{array}{l}\text { aeolian Penrith } \\
\text { Sandstone }\end{array}$ & 1 & l \\
\hline Knust et al., 2009 & $\begin{array}{l}\text { USA; Nevada; Truckee River; Desert } \\
\text { biogeographic. Rainfall: } 18 \mathrm{~cm} / \text { year }\end{array}$ & / & / & coniferous forests \\
\hline $\begin{array}{l}\text { Krause et al., } \\
2013\end{array}$ & $\begin{array}{l}\text { Europe; UK; River Tern; Atlantic } \\
\text { biogeographic }\end{array}$ & Permo-Triassic sandstone & / & / \\
\hline $\begin{array}{l}\text { Laenen and } \\
\text { Bencala, } 2001\end{array}$ & $\begin{array}{l}\text { USA; Oregon;Willamette River; Humid- } \\
\text { semiarid biogeographic }\end{array}$ & late Pleistocene & 1 & 1 \\
\hline $\begin{array}{l}\text { Lamontagne and } \\
\text { Cook, } 2007\end{array}$ & $\begin{array}{l}\text { Australia; Swamp Oak creek; } \\
\text { Subtropical biogeographic region } \\
\text { Rainfall: } 670 \mathrm{~mm} / \mathrm{yr}\end{array}$ & 1 & I & 1 \\
\hline $\begin{array}{l}\text { Lansdown et al., } \\
2012\end{array}$ & $\begin{array}{l}\text { Europe; UK; River Leith;Atlantic } \\
\text { biogeographic }\end{array}$ & $\begin{array}{l}\text { Permo-Triassic } \\
\text { sandstone }\end{array}$ & 1 & \\
\hline $\begin{array}{l}\text { Lautz and Siegel, } \\
2006 \text { Lautz and } \\
\text { Siegel, } 2007\end{array}$ & $\begin{array}{l}\text { USA; Wyoming; Red Canyon Creek; } \\
\text { Semi-arid Intermountain province. } \\
\text { Rainfall: } 35 \mathrm{~cm} / \mathrm{yr}\end{array}$ & $\begin{array}{l}\text { Phosphoria Formation. } \\
\text { Chugwater Formation. } \\
\text { Gravel terraces }\end{array}$ & 1 & livestock grazing \\
\hline $\begin{array}{l}\text { Malcolm et al., } \\
2005\end{array}$ & $\begin{array}{l}\text { Europe; UK; Glen Girnock; Atlantic } \\
\text { biogeographic regionRainfall: } 1100 \\
\mathrm{~mm} / \mathrm{yr}\end{array}$ & schists and gneisses & 1 & $\begin{array}{l}\text { semi-natural heather (Calluna) } \\
\text { moorland }\end{array}$ \\
\hline $\begin{array}{l}\text { Malcolm et al., } \\
2010\end{array}$ & $\begin{array}{l}\text { Europe; UK ; Newmills Burn Girnock } \\
\text { Burn; Atlantic biogeographic region }\end{array}$ & $\begin{array}{l}\text { psammite and pelite, } \\
\text { granite and schist }\end{array}$ & 1 & arable farming and livestook \\
\hline $\begin{array}{l}\text { Malzone et al., } \\
2015\end{array}$ & $\begin{array}{l}\text { USA; New York State; Elton Creek } \\
\text { Continental biogeographic region } \\
\text { Rainfall: } 760 \text { and } 1145 \mathrm{~mm}\end{array}$ & $\begin{array}{l}\text { Glaciated Appalachian } \\
\text { Plateau }\end{array}$ & 1 & $\begin{array}{l}\text { Land use is primarily forest, dairy, } \\
\text { and agriculture with few urban } \\
\text { regions }\end{array}$ \\
\hline $\begin{array}{l}\text { Morrice et al., } \\
1997\end{array}$ & $\begin{array}{l}\text { USA; New Mexico;Aspen Creek } \\
\text { Calaveras GallinaCreek; Semiarid } \\
\text { biogeographic region }\end{array}$ & $\begin{array}{l}\text { Permian sandstone and } \\
\text { siltstone Bandelier turf } \\
\text { granite/gneiss }\end{array}$ & 1 & l \\
\hline
\end{tabular}




\begin{tabular}{|c|c|c|c|c|}
\hline Mouw et al., 2009 & $\begin{array}{l}\text { Alaska; Middle Flathead River } \\
\text { Talkeetna River; Artic biogeographic } \\
\text { region }\end{array}$ & I & / & I \\
\hline Munz et al., 2011 & $\begin{array}{l}\text { Europe; UK; River Leith; Atlantic } \\
\text { biogeographic region Rainfall: } 900 \\
\text { mm/yr }\end{array}$ & Permotriassic Sandstone & 1 & 1 \\
\hline Mutz et al., 2000 & $\begin{array}{l}\text { Europe; Germany; Schlaube Stream; } \\
\text { Continental biogeographic region }\end{array}$ & 1 & 1 & 1 \\
\hline Mutz et al., 2003 & $\begin{array}{l}\text { Europe; Germany; Schlaube Stream; } \\
\text { Continental biogeographic region }\end{array}$ & 1 & & 1 \\
\hline $\begin{array}{l}\text { O'Connor et al., } \\
2008\end{array}$ & $\begin{array}{l}\text { USA; California; Elder Creek; } \\
\text { Mediterranean biogeographic region }\end{array}$ & I & 1 & 1 \\
\hline Ock et al., 2015 & $\begin{array}{l}\text { USA; California; The Trinity River; } \\
\text { Mediterranean biogeographic region }\end{array}$ & 1 & 1 & $\begin{array}{l}\text { Impounded by the Trinity Dam and } \\
\text { the Lewiston Dam since } 1964 \text {. Flow } \\
\text { diverted into the Sacramento River } \\
\text { for field irrigation }\end{array}$ \\
\hline Pinay et al., 2009 & $\begin{array}{l}\text { Alaska; Lynx Creek; Artic } \\
\text { biogeographic region }\end{array}$ & 1 & & $\begin{array}{l}\text { boreal forest association of white } \\
\text { spruce, Picea glauca, interspersed } \\
\text { with balsam poplar, Populus } \\
\text { balsamifera }\end{array}$ \\
\hline Ruehl et al., 2006 & $\begin{array}{l}\text { USA; California; Pajaro River; } \\
\text { Mediterranean biogeographic region. } \\
\text { Rainfall: } 33-55 \mathrm{~cm} / \mathrm{yr}\end{array}$ & $\begin{array}{l}\text { Holocene deposits, the } \\
\text { Aromas Formation } \\
\text { (Pleistocene), Purisima } \\
\text { Formation (Pliocene) } \\
\end{array}$ & 1 & agriculture land use \\
\hline $\begin{array}{l}\text { Sawyer et al., } \\
2012\end{array}$ & $\begin{array}{l}\text { USA; New Mexico; San Antonio } \\
\text { Creek; Semiarid biogeographic region } \\
\text { Rainfall: } 476 \mathrm{~mm} / \mathrm{yr}\end{array}$ & l & 1 & 1 \\
\hline $\begin{array}{l}\text { Stofleth et al. } \\
2008\end{array}$ & $\begin{array}{l}\text { USA; Mississippi; Topashaw Creek; } \\
\text { Continental biogeographic region }\end{array}$ & I & 1 & 1 \\
\hline $\begin{array}{l}\text { Stonedahl et al. , } \\
2012\end{array}$ & $\begin{array}{l}\text { USA; Indiana; Sugar Creek; } \\
\text { Continental biogeographic region }\end{array}$ & I & 1 & 1 \\
\hline $\begin{array}{l}\text { Storey et al. } \\
2003\end{array}$ & $\begin{array}{l}\text { Canada; Ontario; Speed } \\
\text { RiverContinental biogeographic region }\end{array}$ & $\begin{array}{l}\text { primary aquifer is in the } \\
\text { dolomite bedrock. The } \\
\text { bedrock is overlain by } \\
\text { layers of low-permeability }\end{array}$ & 1 & 1 \\
\hline
\end{tabular}




\begin{tabular}{|c|c|c|c|c|}
\hline & & $\begin{array}{l}\text { glacial till, kame, and } \\
\text { outwash deposits }\end{array}$ & & \\
\hline $\begin{array}{l}\text { Swanson et al. } \\
2010\end{array}$ & $\begin{array}{l}\text { USA; New Mexico; Jaramillo Creek; } \\
\text { Semiarid biogeographic region }\end{array}$ & I & 1 & 1 \\
\hline $\begin{array}{l}\text { Thomas et al. } \\
2003\end{array}$ & $\begin{array}{l}\text { USA; North Carolina; Snake Den } \\
\text { Branch; Continental biogeographic } \\
\text { region; Rainfall: } 200 \mathrm{~cm} / \mathrm{yr}\end{array}$ & $\begin{array}{l}\text { crystalline rock, schists, } \\
\text { gneiss }\end{array}$ & 1 & 1 \\
\hline $\begin{array}{l}\text { Triska et al. , } \\
1993\end{array}$ & $\begin{array}{l}\text { USA; California; Little Lost Man Creek; } \\
\text { Continental biogeographic region }\end{array}$ & 1 & 1 & 1 \\
\hline $\begin{array}{l}\text { Wagenhoff et al. } \\
2014\end{array}$ & $\begin{array}{l}\text { New Zealand; Kiripaka Stream } \\
\text { Whakakai Stream; Atlantic } \\
\text { biogeographic region }\end{array}$ & $\begin{array}{l}\text { sedimentary sandstones } \\
\text { and siltstones }\end{array}$ & 1 & $\begin{array}{l}\text { Kiripaka Stream native forest in the } \\
\text { headwaters and intensive pasture } \\
\text { grazed by sheep and cattle. } \\
\text { Whakakai Stream evergreen } \\
\text { podocarp- hardwood forest }\end{array}$ \\
\hline $\begin{array}{l}\text { Wagner et al. , } \\
2003\end{array}$ & $\begin{array}{l}\text { Europe; Austria; Oberer Seebach; } \\
\text { Continental biogeographic region }\end{array}$ & 1 & 1 & l \\
\hline $\begin{array}{l}\text { Wondzell et al. } \\
2009\end{array}$ & $\begin{array}{l}\text { USA; Alaska; Bambi Creek; Artic } \\
\text { biogeographic region. Rainfall: } 1600 \\
\text { mm/a }\end{array}$ & I & 1 & 1 \\
\hline $\begin{array}{l}\text { Wondzell et al. } \\
2006\end{array}$ & $\begin{array}{l}\text { USA; Oregon Andrews Experimental } \\
\text { Forest; Humid-semiarid biogeographic } \\
\text { region }\end{array}$ & bedrock outcrops & 1 & 1 \\
\hline $\begin{array}{l}\text { Wroblicky et al. } \\
1998\end{array}$ & $\begin{array}{l}\text { USA; New Mexico; Aspen Creek, Rio } \\
\text { Calavera; semiarid biogeographic } \\
\text { region }\end{array}$ & $\begin{array}{l}\text { Lower Permian fine } \\
\text { sandstones and siltstones } \\
\text { of the Meseta Blanca } \\
\text { Member of the Lower } \\
\text { Yeso Formation }\end{array}$ & I & I \\
\hline $\begin{array}{l}\text { Zarnetske et al. , } \\
2011\end{array}$ & $\begin{array}{l}\text { USA; Oregon; Drift Creek; humid- } \\
\text { semiarid biogeographic region. } \\
\text { Rainfall: } 1190 \mathrm{~mm} / \mathrm{yr}\end{array}$ & I & I & $\begin{array}{l}\text { agriculture (lower catchment) } \\
\text { forestry (upper catchment) }\end{array}$ \\
\hline
\end{tabular}


Table s2: Collection of worldwide field studies on the hyporheic exchange across a broad range of hydrogeological, topographical and geological settings. Table shows references, geographical zone and state, river name, and information on valley scale: geology (sediment size and hydraulic conductivity), topographical/morphological, ecological (in-channel vegetation), anthropogenic (agricultural or human infrastructure).

\begin{tabular}{|c|c|c|c|c|c|}
\hline Source & Hydrological & Hydrogeological & Topographical & Ecological & Anthropogenic \\
\hline $\begin{array}{l}\text { Angermann et } \\
\text { al., } 2012\end{array}$ & $\begin{array}{l}\text { Mean discharge is } 0.9 \mathrm{~m} 3 \mathrm{~s}- \\
1 \text { with Q95 of } 0.4 \mathrm{~m} 3 \mathrm{~s}-1 \text { and } \\
\text { Q10 of } 1.39 \mathrm{~m} 3 \mathrm{~s}-1\end{array}$ & I & lowland & / & agricultural \\
\hline $\begin{array}{l}\text { Anibas et al., } \\
2012\end{array}$ & $\begin{array}{l}\text { regular flood event after } \\
\text { snowmelt in the upper } \\
\text { catchment peatland is } \\
\text { mostly groundwtaer fed }\end{array}$ & $\begin{array}{l}\text { The morainic plateau } \\
\text { is composed of } \\
\text { heterogeneous loamy } \\
\text { sand deposits. The flat } \\
\text { alluvial valleys are } \\
\text { filled with thick } \\
\text { deposits of } \\
\text { fluvioglacial sand and } \\
\text { gravel which are } \\
\text { covered by a variety of } \\
\text { organic soils. }\end{array}$ & $\begin{array}{l}\text { Lowland mashes and } \\
\text { peat lenses }(2-5 \mathrm{~cm})\end{array}$ & $\begin{array}{l}\text { oak-beech } \\
\text { forests,reed } \\
\text { vegetation in the } \\
\text { center of the valley } \\
\text { and sedges closer to } \\
\text { the slope crack }\end{array}$ & $\begin{array}{l}\text { arable lands meandow } \\
\text { and pastures in the lower } \\
\text { section of the catchment }\end{array}$ \\
\hline $\begin{array}{l}\text { Anibas et al., } \\
2009\end{array}$ & I & I & lowland & I & Agricultural landuse \\
\hline $\begin{array}{l}\text { Arntzen et al., } \\
2006\end{array}$ & gaining condition & unconfined aquifer & various topography & I & $/$ \\
\hline $\begin{array}{l}\text { Bourke et al., } \\
2014\end{array}$ & losing & I & lowland & I & I \\
\hline $\begin{array}{l}\text { Briggs et al., } \\
2010\end{array}$ & I & I & $\mathrm{med} /$ low gradient & I & $\begin{array}{l}\text { wetland, agricultural land, } \\
\text { woodland and urban } \\
\text { areas }\end{array}$ \\
\hline $\begin{array}{l}\text { Czernuszenko } \\
\text { et. al., } 1998\end{array}$ & I & I & lowland & I & 1 \\
\hline $\begin{array}{l}\text { Datry et al } \\
2008\end{array}$ & $\begin{array}{l}\text { larger gaining and } \\
\text { losing sections of the valley }\end{array}$ & / & & shrubs & / \\
\hline Dujardin et al & / & I & lowland & I & / \\
\hline
\end{tabular}




\begin{tabular}{|c|c|c|c|c|c|}
\hline \multicolumn{6}{|l|}{2014} \\
\hline $\begin{array}{l}\text { Duke et al } \\
2007\end{array}$ & I & I & 1 & $\begin{array}{l}\text { Riparian forest: } \\
\text { Ulmus crassifolia, } \\
\text { Fraxinus } \\
\text { texensis, Juniperus } \\
\text { ashei }\end{array}$ & agriculture, small \\
\hline $\begin{array}{l}\text { Edwardson et } \\
\text { al., } 2003\end{array}$ & I & I & braided, sinuous & $\begin{array}{l}\text { Carex aquatilis and } \\
\text { Eriophorum } \\
\text { vaginatum, Betula } \\
\text { nana }\end{array}$ & \\
\hline $\begin{array}{l}\text { Haggard et al., } \\
2001\end{array}$ & 1 & shallow silt loams. & I & $\begin{array}{l}\text { sycamore trees in } \\
\text { Dry Creek and a mix } \\
\text { of sycamore trees } \\
\text { and other hardwoods } \\
\text { in Cloud Creek and } \\
\text { Cherokee Creek. }\end{array}$ & $\begin{array}{l}\text { Dry Creek and Cherokee } \\
\text { Creek had large grass } \\
\text { pastures whereas the } \\
\text { up-slope vegetation at } \\
\text { Cloud Creek was } \\
\text { characterized by } \\
\text { underbrush and forest } \\
\text { layers }\end{array}$ \\
\hline Hall et al., 2002 & I & I & medium/low gradient & $\begin{array}{l}\text { American beech } \\
\text { sugar maple yellow } \\
\text { birch }\end{array}$ & \\
\hline $\begin{array}{l}\text { Hart et el., } \\
1999\end{array}$ & I & I & I & deciduous forest & \\
\hline $\begin{array}{l}\text { Harvey and } \\
\text { Fuller, } 1998 \\
\text { Harvey et al. , } \\
2003\end{array}$ & I & I & medium/low gradient & $\begin{array}{l}\text { tamarisk seedlings, } \\
\text { willows }\end{array}$ & \\
\hline $\begin{array}{l}\text { Jones et al. , } \\
2008\end{array}$ & I & I & $\begin{array}{l}\text { bedrock valley with } \\
\text { spring }\end{array}$ & I & I \\
\hline $\begin{array}{l}\text { Kasahara et } \\
\text { al., } 2003\end{array}$ & 1 & I & $\begin{array}{l}\text { Upland bedrock } \\
\text { contrained and } \\
\text { unconstrained sections }\end{array}$ & & I \\
\hline $\begin{array}{l}\text { Kasahara et al. } \\
, 2006\end{array}$ & I & I & lowland & $\begin{array}{l}\text { grass-vegetated } \\
\text { floodplain }\end{array}$ & $\begin{array}{l}\text { residential and } \\
\text { agriculture fields }\end{array}$ \\
\hline
\end{tabular}




\begin{tabular}{|c|c|c|c|c|c|}
\hline $\begin{array}{l}\text { Kasahara et } \\
\text { al., } 2007\end{array}$ & / & l & l & $\begin{array}{l}\text { willows along the } \\
\text { banks }\end{array}$ & $\begin{array}{l}\text { agricultural (crop and } \\
\text { soya beans) }\end{array}$ \\
\hline $\begin{array}{l}\text { Kaser et al., } \\
2013\end{array}$ & gaining & 1 & $\begin{array}{l}\text { meanders within a } \\
\text { narrow floodplain } \\
(<100)\end{array}$ & I & I \\
\hline $\begin{array}{l}\text { Knust et al., } \\
2009\end{array}$ & I & I & lowland & coniferous forest & 1 \\
\hline $\begin{array}{l}\text { Krause et al., } \\
2013\end{array}$ & 1 & I & lowland & I & $\begin{array}{l}\text { extensive agricultural } \\
\text { land use }\end{array}$ \\
\hline $\begin{array}{l}\text { Laenen and } \\
\text { Bencala, } 2001\end{array}$ & 1 & 1 & Lowland alluvial fans & I & l \\
\hline $\begin{array}{l}\text { Lamontagne } \\
\text { and Cook, } \\
2007\end{array}$ & I & I & & / & I \\
\hline $\begin{array}{l}\text { Lansdown et } \\
\text { al., } 2012\end{array}$ & I & 1 & lowland & I & agricultural fields \\
\hline $\begin{array}{l}\text { Lautz and } \\
\text { Siegel, } 2006 \\
\text { Lautz and } \\
\text { Siegel, } 2007\end{array}$ & I & I & upland & l & l \\
\hline $\begin{array}{l}\text { Malcolm et al., } \\
2005\end{array}$ & $\begin{array}{l}\text { mean discharge of } 0.5 \mathrm{~m} 3 \mathrm{~s}- \\
1, \text { varying between }<0.01 \\
\mathrm{~m} 3 \mathrm{~s}-1 \text { in the summer and } \\
>23 \mathrm{~m} 3 \mathrm{~s}-1 \text { during floods }\end{array}$ & I & upland & $\begin{array}{l}\text { heather (Calluna) } \\
\text { moorland }\end{array}$ & ( \\
\hline $\begin{array}{l}\text { Malcolm et al., } \\
2010\end{array}$ & l & I & lowland & l & $\begin{array}{l}\text { heather moorland, and } \\
\text { commercial and } \\
\text { semi-natural forest in the } \\
\text { lower catchment }\end{array}$ \\
\hline $\begin{array}{l}\text { Malzone et al., } \\
2015\end{array}$ & gaining & 1 & gaining stream & I & l \\
\hline $\begin{array}{l}\text { Morrice et al., } \\
1997\end{array}$ & 1 & I & upland & I & I \\
\hline
\end{tabular}




\begin{tabular}{|c|c|c|c|c|c|}
\hline $\begin{array}{l}\text { Mouw et al., } \\
2009\end{array}$ & I & I & $\begin{array}{l}\text { lowland. Large alluvial } \\
\text { flood plains }\end{array}$ & $\begin{array}{l}\text { Populus balsamifera } \\
\text { and shrub } \\
\text { communities by Salix } \\
\text { sp and Alnus incana } \\
\text { on surfaces flooded. } \\
\text { Forested benches } \\
\text { are dominated by } P \text {. } \\
\text { balsamifera, Picea } \\
\text { engelmannii, } \\
\text { Pseudotsuga } \\
\text { menziesii, Larix } \\
\text { occidentalis,Abies } \\
\text { lasiocarpa }\end{array}$ & $\begin{array}{l}\text { cottonwood and spruce } \\
\text { forests }\end{array}$ \\
\hline $\begin{array}{l}\text { Munz et al., } \\
2011\end{array}$ & I & 1 & $\begin{array}{l}\text { lowland. narrow } \\
\text { floodplain } 100 \mathrm{~m} \text { wide. } \\
\text { steep slopes with } \\
\text { occasional outcrops of } \\
\text { the PTS bedrock }\end{array}$ & grassland vegetation & livestock grazing \\
\hline $\begin{array}{l}\text { Mutz et al., } \\
2000\end{array}$ & I & I & $\begin{array}{l}\text { lowland. many springs } \\
\text { and small streamlets } \\
\text { draining from the valley } \\
\text { slopes into the stream }\end{array}$ & I & I \\
\hline $\begin{array}{l}\text { Mutz et al., } \\
2003\end{array}$ & I & I & lowland & woodland & I \\
\hline $\begin{array}{l}\text { O'Connor et } \\
\text { al., } 2008\end{array}$ & I & I & lowland & I & I \\
\hline $\begin{array}{l}\text { Pinay et al., } \\
2009\end{array}$ & I & 1 & I & $\begin{array}{l}\text { willow Salix sp.,moist } \\
\text { tundra communities } \\
\text { at low elevations; } \\
\text { and extensive stands } \\
\text { of green alder, Alnus } \\
\text { crispa, at higher } \\
\text { elevations. Little } \\
\text { alder is found in } \\
\text { riparian areas }\end{array}$ & 1 \\
\hline
\end{tabular}




\begin{tabular}{|c|c|c|c|c|c|}
\hline $\begin{array}{l}\text { Ruehl et al., } \\
2006\end{array}$ & $\begin{array}{l}\text { mean daily discharge } \\
0 \text { and } 610 \mathrm{~m} 3 / \mathrm{s} \text { from } 1939 \\
\text { to } 2003 .\end{array}$ & / & lowland & / & / \\
\hline $\begin{array}{l}\text { Sawyer et al., } \\
2012\end{array}$ & I & I & $\begin{array}{l}\text { lowland, unconfined } \\
\text { valley }\end{array}$ & / & 1 \\
\hline $\begin{array}{l}\text { Stofleth et al. , } \\
2008\end{array}$ & I & I & $\begin{array}{l}\text { lowland, straightened } \\
\text { (upstream) and } \\
\text { channelized } \\
\text { (downstream) the } \\
\text { chosen reach }\end{array}$ & / & $\begin{array}{l}\text { Little Topashaw Creek: } \\
\text { cultivated valley floors } \\
\text { and forested hillslopes } \\
\text { Goodwin Creek } \\
\text { predominately is forest, } \\
\text { pasture, and fallow lands }\end{array}$ \\
\hline $\begin{array}{l}\text { Stonedahl et al. } \\
\text {, } 2012\end{array}$ & I & I & $\begin{array}{l}\text { Lowland ditched and } \\
\text { straightened }\end{array}$ & 1 & agricultural fields \\
\hline $\begin{array}{l}\text { Storey et al. , } \\
2003\end{array}$ & I & / & lowland & / & / \\
\hline $\begin{array}{l}\text { Swanson et al. } \\
, 2010\end{array}$ & I & I & $\begin{array}{l}\text { sinuous, with steep } \\
\text { stream banks }\end{array}$ & 1 & 1 \\
\hline $\begin{array}{l}\text { Thomas et al. , } \\
2003\end{array}$ & I & I & steep & $\begin{array}{l}\text { mountain laurel } \\
\text { (Kalmia latifolia L.) } \\
\text { and rhododendron } \\
\text { (Rhododendron } \\
\text { maximum L.) } \\
\end{array}$ & I \\
\hline $\begin{array}{l}\text { Wagenhoff et } \\
\text { al. , } 2014\end{array}$ & I & I & upland & I & 1 \\
\hline $\begin{array}{l}\text { Wagner et al. , } \\
2003\end{array}$ & I & I & alpine & I & I \\
\hline $\begin{array}{l}\text { Wondzell et al. } \\
\text {, } 2009\end{array}$ & 1 & 1 & lowland & 1 & natural \\
\hline $\begin{array}{l}\text { Wondzell et al. } \\
, 2006\end{array}$ & I & I & unconstrained & / & 1 \\
\hline $\begin{array}{l}\text { Wroblicky et al. } \\
, 1998\end{array}$ & 1 & 1 & variable & 1 & 1 \\
\hline
\end{tabular}


Table S3: Collection of worldwide field studies on the hyporheic exchange across a broad range of hydrogeological, topographical and geological settings. Table shows references, geographical zone and state, river name, and information on reach: geology (sediment size and hydraulic conductivity), topographical/morphological, ecological (in-channel vegetation), anthropogenic (agricultural or human infrastructure).

\begin{tabular}{|c|c|c|c|c|}
\hline Source & Hydrological & Hydrogeological & Topographical & Ecological \\
\hline $\begin{array}{l}\text { Angermann et al., } \\
2012\end{array}$ & $\begin{array}{l}\text { midsize gravel,different sizes of sand, fine silty } \\
\text { materials. }\end{array}$ & $\begin{array}{l}\text { hydraulic } \\
\text { conductivities: } 10^{-3} \text { to } \\
10^{-5} \mathrm{~ms}^{-1} \text { and } 10^{-8} \text { to } \\
10^{-9} \mathrm{~ms}^{-1} \text { (Krause et al., } \\
2012 \text { ) }\end{array}$ & $\begin{array}{l}\text { Meander, pool- } \\
\text { riffle- pool } \\
\text { bedforms }\end{array}$ & / \\
\hline $\begin{array}{l}\text { Anibas et al., } \\
2012\end{array}$ & $\begin{array}{l}\text { loamy sand deposits; thick deposits of fluvioglacial } \\
\text { sands and gravels which are covered by a variety } \\
\text { of organic soil. }\end{array}$ & $\begin{array}{l}\text { see Table } 2 \text { for } \\
\text { hydraulic conductivities }\end{array}$ & $\begin{array}{l}\text { meander and } \\
\text { straight section } \\
\text { steep banks }\end{array}$ & $\begin{array}{l}\text { banks mostly are covered } \\
\text { with reed plants. }\end{array}$ \\
\hline $\begin{array}{l}\text { Anibas et al., } \\
2009\end{array}$ & fine sand and some organic material & $\begin{array}{l}\text { thermal conductivity: } \\
1.8 \mathrm{Js}^{-1} \mathrm{~m}^{-1} \mathrm{~K}^{-1}\end{array}$ & $\begin{array}{l}\text { straight and } \\
\text { canalized }\end{array}$ & / \\
\hline $\begin{array}{l}\text { Arntzen et al., } \\
2006\end{array}$ & $\begin{array}{l}\text { cobble }(>64 \text { to }<=128 \mathrm{~mm} \text { ) in a matrix of fine sand } \\
(>0.062 \text { to }<=0.5 \mathrm{~mm}) . \text { At } \mathrm{rkm} 602 \text { site, the median } \\
\text { grain size-D50 was } 57.7 \mathrm{~mm} \text {. At rkm } 582 \text { site, the } \\
\text { dominant substrate was coarse gravel ( }>16 \\
\text { to }<=64 \mathrm{~mm} \text { ) in a matrix consisting mostly of fine } \\
\text { sand }(>0.062 \text { to }<-=0.5 \mathrm{~mm}) \text {. The D50 at rkm } 582 \\
\text { was } 35.5 \mathrm{~mm} \text {. At rkm } 577 \text {, the dominant substrate } \\
\text { was coarse gravel }(>16 \text { to }<=64 \mathrm{~mm} \text { ) in a matrix } \\
\text { consisting of fine sand. However, there was a silt } \\
\text { component at rkm } 577 \text { much larger than at the } \\
\text { other two locations. The D50 at rkm } 577 \text { was } \\
22.3 \mathrm{~mm} \text {. }\end{array}$ & $\begin{array}{l}\text { hydraulic } \\
\text { conductivities: } 8.8 \times 10^{-3} \\
\mathrm{cms}^{-1} \text { to } 2.9 \times 10^{-4} \mathrm{cms}^{-1}\end{array}$ & I & / \\
\hline $\begin{array}{l}\text { Bourke et al., } \\
2014\end{array}$ & / & $\begin{array}{l}\text { hydraulic } \\
\text { conductivity } 1500 \text { and } \\
3700 \mathrm{md}^{-1}\end{array}$ & $\begin{array}{l}\text { straight, section } \\
\text { with pools, riffles } \\
\text { and glides } \\
\text { bedforms }\end{array}$ & / \\
\hline Briggs et al., 2010 & hallow soils and glacial deposits & I & meander section & $\begin{array}{l}\text { beaver activity and wood } \\
\text { dams }\end{array}$ \\
\hline
\end{tabular}




\begin{tabular}{|c|c|c|c|c|}
\hline $\begin{array}{l}\text { Czernuszenko et. } \\
\text { al., } 1998\end{array}$ & / & / & $\begin{array}{l}\text { Straight Low } \\
\text { sinuosity }\end{array}$ & / \\
\hline Datry et al 2008 & gravels, cobbles, and small boulders & / & $\begin{array}{l}\text { riffles, prafluvial } \\
\text { bars, terraces }\end{array}$ & annual grasses and herbs \\
\hline $\begin{array}{l}\text { Dujardin et al } \\
2014\end{array}$ & silty and clay- loam & $\begin{array}{l}\text { hydraulic conductivities } \\
\text { see Table } 2\end{array}$ & / & / \\
\hline Duke et al 2007 & clay-rich vertisol & $0.104 \mathrm{~cm} \mathrm{~h}^{-1}$ & / & J. ashei \\
\hline $\begin{array}{l}\text { Edwardson et al., } \\
2003\end{array}$ & cobble, gravel,peat but variable according to site & $\begin{array}{l}\text { hydraulic } \\
\text { conductivities: see } \\
\text { Table } 3\end{array}$ & $\begin{array}{l}\text { pool riffles, } \\
\text { meanders,Debris } \\
\text { dam }\end{array}$ & / \\
\hline $\begin{array}{l}\text { Fernald et al., } \\
2001\end{array}$ & gravel Holocene deposit & $10^{-2}$ and $10^{-1} \mathrm{~m} \mathrm{~s}^{-1}$ & $\begin{array}{l}\text { two large island } \\
\text { complexes with } \\
\text { anastomosing } \\
\text { channels and } \\
\text { extensive exposed } \\
\text { gravels. }\end{array}$ & / \\
\hline $\begin{array}{l}\text { Gooseff et al., } \\
2003\end{array}$ & very porous & / & 1 & / \\
\hline $\begin{array}{l}\text { Haggard et al., } \\
2001\end{array}$ & cobbles with some fines & / & / & I \\
\hline Hall et al., 2002 & cobbles and boulders & / & debris dams & / \\
\hline Hart et el., 1999 & gravel and cobbles, bedrock outcrops & / & $\begin{array}{l}\text { boulders and } \\
\text { debris dams are }\end{array}$ & $\begin{array}{l}\text { imput of deciduous forest: } \\
\text { leaves }\end{array}$ \\
\hline $\begin{array}{l}\text { Harvey and Fuller, } \\
\text { 1998, Harvey et } \\
\text { al. , } 2003\end{array}$ & sand and gravel & 1 & $\begin{array}{l}\text { straight section } \\
\text { cobbles and } \\
\text { channel parallel } \\
\text { bars at side and } \\
\text { central channel }\end{array}$ & / \\
\hline Jones et al. , 2008 & $\begin{array}{l}\text { basalt gravel, cobbles, and boulders intermixed } \\
\text { with silt and sand lenses }\end{array}$ & 300 to $700 \mathrm{~m} \mathrm{day}^{-1}$ & $\begin{array}{l}\text { naturally } \\
\text { anabranched }\end{array}$ & $\begin{array}{l}\text { in-channel macrophytes } \\
\text { vegetation (season } \\
\text { dependent) }\end{array}$ \\
\hline $\begin{array}{l}\text { Kasahara et al., } \\
2003\end{array}$ & coarse-textured gravel & 1 & 1 & 1 \\
\hline
\end{tabular}




\begin{tabular}{|c|c|c|c|c|}
\hline $\begin{array}{l}\text { Kasahara et al. , } \\
2006\end{array}$ & cobbles & $\begin{array}{l}<0.1 \mathrm{~m} \text { day }^{-1} \text { to }>10 \mathrm{~m} \\
\text { day }^{-1}\end{array}$ & $\begin{array}{l}\text { riffles and step } \\
\text { from restoration } \\
\text { project }\end{array}$ & / \\
\hline $\begin{array}{l}\text { Kasahara et al., } \\
2007\end{array}$ & gravel bed channel,silt and clay & 0.3 to $>20 \mathrm{~m} \mathrm{day}^{-1}$ & $\begin{array}{l}\text { gravel bar and } \\
\text { menader bends } \\
\text { were selected for } \\
\text { studying }\end{array}$ & \\
\hline Kaser et al., 2013 & soft sediment & $2.7-2.8 \times 10^{-5} \mathrm{~ms}^{-1}$ & $\begin{array}{l}\text { Meander riffle-pool } \\
\text { sequences }\end{array}$ & / \\
\hline Knust et al., 2009 & cobbles and boulders & / & $\begin{array}{l}\text { straighter and } \\
\text { wider }\end{array}$ & / \\
\hline $\begin{array}{l}\text { Krause et al., } \\
2013\end{array}$ & $\begin{array}{l}\text { Midsize gravel, Different sizes of sand Fine silty } \\
\text { materials }\end{array}$ & $\begin{array}{l}\text { hydraulic conductivities } \\
10^{-3} \text { to } 10^{-5} \mathrm{~ms}^{-1}\end{array}$ & $\begin{array}{l}\text { meandering } \\
\text { section steep river } \\
\text { banks, pool-riffle- } \\
\text { pool sequences }\end{array}$ & / \\
\hline $\begin{array}{l}\text { Laenen and } \\
\text { Bencala, } 2001\end{array}$ & sands, silts, and clays & / & $\begin{array}{l}\text { meandering and } \\
\text { braided channel } \\
\text { with many islands } \\
\text { and sloughs. }\end{array}$ & 1 \\
\hline $\begin{array}{l}\text { Lamontagne and } \\
\text { Cook, } 2007\end{array}$ & coarse sand, gravel, and cobble & porosity of $\sim 0.4$ & I & / \\
\hline $\begin{array}{l}\text { Lansdown et al., } \\
2012\end{array}$ & sand, gravel, and cobbles on sands and silts & / & $\begin{array}{l}\text { riffle and pool } \\
\text { sequences }\end{array}$ & 1 \\
\hline $\begin{array}{l}\text { Lautz and Siegel, } \\
\text { 2006, Lautz and } \\
\text { Siegel, } 2007\end{array}$ & gravel and fine sand but also silt & $\begin{array}{l}\text { hydraulic conductivity } \\
\text { see Table } 1 \text { Lautz and } \\
\text { Siegel } 2006\end{array}$ & $\begin{array}{l}\text { meandering and } \\
\text { straight sections }\end{array}$ & $\begin{array}{l}\text { debris dams and small log } \\
\text { dams (natural and non) }\end{array}$ \\
\hline $\begin{array}{l}\text { Malcolm et al., } \\
2005\end{array}$ & Podzols, gleys and peats & 1 & / & 1 \\
\hline $\begin{array}{l}\text { Malcolm et al., } \\
2010\end{array}$ & $\begin{array}{l}\text { overlain by glacial till and meltwater deposits and } \\
\text { overlain by glacial and fluvioglacial deposits. }\end{array}$ & / & $\begin{array}{l}\text { deepened and } \\
\text { straightened pool } \\
\text { riffles,bars }\end{array}$ & / \\
\hline $\begin{array}{l}\text { Malzone et al., } \\
2015\end{array}$ & sand, gravel, clay, and till & / & $\begin{array}{l}\text { pool and riffles } \\
\text { sequences }\end{array}$ & / \\
\hline
\end{tabular}




\begin{tabular}{|c|c|c|c|c|}
\hline $\begin{array}{l}\text { Morrice et al., } \\
1997\end{array}$ & $\begin{array}{l}2 \% \text { gravel } 46 \% \text { sand } 42 \% \text { silt } 10 \% \text { clay } 36 \% \text { gravel } \\
53 \% \text { sand } 9 \% \text { silt } 2 \% \text { clay boulders cobbles gravel } \\
\text { and sand }\end{array}$ & | 1 & / & / \\
\hline Mouw et al., 2009 & gravel to sand & / & $\begin{array}{l}\text { anastomosing } \\
\text { channels pools }\end{array}$ & / \\
\hline Munz et al., 2011 & silt to coarse sand & / & $\begin{array}{l}\text { meandering } \\
\text { section of the river. } \\
\text { Longitudinal pool- } \\
\text { riffle-pool } \\
\text { sequence }\end{array}$ & $\begin{array}{l}\text { riparian reed and grass } \\
\text { vegetation. wet grassland } \\
\text { and sparse riparian soft- } \\
\text { wood vegetation alongside }\end{array}$ \\
\hline Mutz et al., 2000 & Fine/ medium sand with some gravel & / & sinuous & $\begin{array}{l}\text { Alnus glutinosa and } \\
\text { Carpinus petulus. In- } \\
\text { channel wood. }\end{array}$ \\
\hline Mutz et al., 2003 & coarse to fine sands & 1 & / & 1 \\
\hline $\begin{array}{l}\text { O'Connor et al., } \\
2008\end{array}$ & boulders & / & $\begin{array}{l}\text { riffle-pool. slope of } \\
0.026 \text {. sinuosity } \\
\text { ratio of } 1.1\end{array}$ & / \\
\hline Ock et al., 2015 & coarse gravel & ( & $\begin{array}{l}4 \text { channel-gravel } \\
\text { features (bars and } \\
\text { vegetated islands) }\end{array}$ & / \\
\hline Pinay et al., 2009 & gravel & $2 \times 10^{-4}$ and $3 \times 10^{-3} \mathrm{cms}^{-1}$ & I & / \\
\hline Ruehl et al., 2006 & / & & / & / \\
\hline $\begin{array}{l}\text { Sawyer et al., } \\
2012\end{array}$ & Mix cobbles, gravel on silt & $4.0 \mathrm{~m} \mathrm{~d}^{-1}$ & $\begin{array}{l}\text { two straight runs } \\
\text { separated by a } \\
\text { meander. Pool and } \\
\text { riffle sequences }\end{array}$ & grasses and forbs. \\
\hline $\begin{array}{l}\text { Stofleth et al. , } \\
2008\end{array}$ & silt and clay soils over sand & 1 & $\begin{array}{l}\text { tortuous reach } \\
\text { section mild } \\
\text { channel slope }\end{array}$ & / \\
\hline $\begin{array}{l}\text { Stonedahl et al. , } \\
2012\end{array}$ & gravel, pebble, and coarse sandy glacial & 1 & pools and riffles & / \\
\hline
\end{tabular}




\begin{tabular}{|c|c|c|c|c|}
\hline $\begin{array}{l}\text { Storey et al. } \\
2003\end{array}$ & recent alluvium & $2 \times 10^{-4} \mathrm{~ms}^{-1}$ & $1 /$ & l \\
\hline $\begin{array}{l}\text { Swanson et al. , } \\
2010\end{array}$ & sand and gravel & $3.2 \times 10^{-5}$ & $\begin{array}{l}\text { losing condition of } \\
\text { the reach and pool- } \\
\text { riffle- pool } \\
\text { sequence }\end{array}$ & $\begin{array}{l}\text { banks stabilized by dense } \\
\text { communities of grasses }\end{array}$ \\
\hline $\begin{array}{l}\text { Thomas et al. , } \\
2003\end{array}$ & colluvial sediments coarse material & 1 & $\begin{array}{l}\text { steep colluvial } \\
\text { sections }\end{array}$ & $\begin{array}{l}\text { large oak-hickory stands } \\
\text { with cove hardwoods } \\
\text { common along the stream } \\
\text { channel }\end{array}$ \\
\hline $\begin{array}{l}\text { Triska et al. } \\
1993\end{array}$ & gravel & 1 & 1 & 1 \\
\hline $\begin{array}{l}\text { Wagenhoff et al. , } \\
2014\end{array}$ & gravels and sand & / & $\begin{array}{l}\text { wood logs in both } \\
\text { streams }\end{array}$ & I \\
\hline $\begin{array}{l}\text { Wagner et al. } \\
2003\end{array}$ & gravel to fine & 1 & $\begin{array}{l}\text { steep slope in the } \\
\text { upstream section. } \\
\text { Downstream } \\
\text { section } \\
\text { characterized by } \\
\text { wetland area } \\
\text { between the hill } \\
\text { and the left bank }\end{array}$ & $\begin{array}{l}\text { Riparian vegetation: Salix } \\
\text { caprea L., Salix myrsinifolia } \\
\text { Picea abies L., Fraxinus } \\
\text { excelsior L., Acer } \\
\text { pseudoplatanus L., Fagus } \\
\text { sylvatica L. and Corylus } \\
\text { avellana L. Also present but } \\
\text { less abundant are Acer } \\
\text { platanoides } L ., \text { Alnus } \\
\text { incana Cornus sanguinea } \\
\text { L. and Crataegus } \\
\text { monogyna }\end{array}$ \\
\hline $\begin{array}{l}\text { Wondzell et al. } \\
2009\end{array}$ & fine gravel to sand & / & low gradient & l \\
\hline $\begin{array}{l}\text { Wondzell et al. } \\
2006\end{array}$ & $\begin{array}{l}\text { boulders, cobbles, gravels and finer textured } \\
\text { sediment }\end{array}$ & $9.2 \mathrm{~m}$ day $^{-1}$ & steep channels & wood debris \\
\hline $\begin{array}{l}\text { Wroblicky et al. } \\
1998\end{array}$ & $\begin{array}{l}\text { poorly sorted, gravelly, coarse sand with } \\
\text { occasional cobbles and boulders. }\end{array}$ & $\begin{array}{l}\text { see Table } 2 \text { for } \\
\text { hydraulic conductivities }\end{array}$ & 1 & I \\
\hline $\begin{array}{l}\text { Zarnetske et al. } \\
2011\end{array}$ & sand, gravel, cobbles, and boulders. & 1 & $\begin{array}{l}\text { planebed and } \\
\text { riffles } 0.007 \mathrm{~m} \mathrm{~m}-1 \\
\text { (reach slope) }\end{array}$ & l \\
\hline
\end{tabular}

